\title{
Criminologie
}

\section{Des expériences affectives et sexuelles en situation de rue}

\section{Philippe-Benoit Côté, Martin Blais, Céline Bellot et Hélène Manseau}

Volume 46, numéro 2, automne 2013

URI : https://id.erudit.org/iderudit/1020995ar

DOI : https://doi.org/10.7202/1020995ar

Aller au sommaire du numéro

Éditeur(s)

Les Presses de l’Université de Montréal

ISSN

0316-0041 (imprimé)

1492-1367 (numérique)

Découvrir la revue

Citer cet article

Côté, P.-B., Blais, M., Bellot, C. \& Manseau, H. (2013). Des expériences affectives et sexuelles en situation de rue. Criminologie, 46(2), 243-261.

https://doi.org/10.7202/1020995ar

\section{Résumé de l'article}

Cette étude a pour objectif de comprendre comment les jeunes construisent leurs expériences affectives et sexuelles en situation de rue à Montréal. Des entrevues individuelles ont été réalisées auprès de 32 jeunes âgés de 18 à 27 ans en situation de rue (18 femmes, 14 hommes). Les témoignages ont été analysés à partir d'une méthode typologique (Schnapper, 2005) qui a permis d'identifier cinq figures d'expériences affectives et sexuelles en situation de rue : la valorisation de la réussite criminelle, le retrait, la survie, l'engagement et l'envahissement par la drogue. L'analyse des témoignages montre que les jeunes des figures du retrait et de l'engagement s'investissent affectivement avec leurs partenaires, tandis que ceux des figures de la valorisation de la réussite criminelle et de la survie décrivent plutôt une instrumentalisation de leurs partenaires. La figure de l'envahissement par la drogue illustre, quant à elle, l'emprise de la consommation de drogues qui pousse les jeunes vers une marchandisation de la sexualité pour répondre à leur dépendance aux substances. Cette étude permet donc de nuancer une conception homogène et menaçante de la situation de rue en faisant voir comment les jeunes mobilisent de diverses façons leurs expériences affectives et sexuelles pour améliorer leur situation de vie.
Ce document est protégé par la loi sur le droit d'auteur. L’utilisation des services d'Érudit (y compris la reproduction) est assujettie à sa politique d'utilisation que vous pouvez consulter en ligne.

https://apropos.erudit.org/fr/usagers/politique-dutilisation/ 


\title{
Des expériences affectives et sexuelles en situation de rue ${ }^{1}$
}

\author{
Philippe-Benoit Côté ${ }^{2}$ \\ Professeur \\ Département de sexologie, Université du Québec à Montréal \\ cote.philippe-benoit@uqam.ca \\ Martin Blais \\ Professeur \\ Département de sexologie, Université du Québec à Montréal, \\ blais.martin@uqam.ca \\ Céline Bellot \\ Professeure \\ École de service social, Université de Montréal \\ celine.bellot@umontreal.ca \\ Hélène Manseau \\ Professeure \\ Département de sexologie, Université du Québec à Montréal \\ manseau.helene@uqam.ca
}

RÉSUMÉ - Cette étude a pour objectif de comprendre comment les jeunes construisent leurs expériences affectives et sexuelles en situation de rue à Montréal. Des entrevues individuelles ont été réalisées auprès de 32 jeunes âgés de 18 à 27 ans en situation de rue (18 femmes, 14 hommes). Les témoignages ont été analysés à partir d'une méthode typologique (Schnapper, 2005) qui a permis d'identifier cinq figures d'expériences affectives et sexuelles en situation de rue: la valorisation de la réussite criminelle, le retrait, la survie, l'engagement et l'envahissement par la drogue. L'analyse des témoignages montre que les jeunes des figures du retrait et de l'engagement s'investissent affectivement avec leurs partenaires, tandis que ceux des figures de la valorisation de la réussite criminelle et de la survie décrivent plutôt une instrumentalisation

1. Les auteurs tiennent à remercier les participants pour leur générosité ainsi que Marie-Andrée Provencher, Michel Martel, Marie Hélène Proulx et Jean-François Truchon pour leur contribution à des phases antérieures de la recherche. Cette recherche a été financée par le Conseil de recherches en sciences humaines.

2. Philippe-Benoit Côté, Département de sexologie, Université du Québec à Montréal, Case postale 8888, succursale Centre-ville, Montréal (Québec), CANADA, H3C 3P8

Criminologie, vol. 46, n 2 (2013) 
de leurs partenaires. La figure de l'envahissement par la drogue illustre, quant à elle, l'emprise de la consommation de drogues qui pousse les jeunes vers une marchandisation de la sexualité pour répondre à leur dépendance aux substances. Cette étude permet donc de nuancer une conception homogène et menaçante de la situation de rue en faisant voir comment les jeunes mobilisent de diverses façons leurs expériences affectives et sexuelles pour améliorer leur situation de vie.

MOTS-CLÉS - Jeunes de la rue, amour, sexualité, typologie, recherche qualitative.

\section{Introduction}

Plusieurs travaux empiriques sur les expériences affectives et sexuelles ${ }^{3}$ des jeunes en situation de rue ${ }^{4}$ tendent à montrer qu'elles présentent d'importants risques pour leur santé. Ces études se concentrent principalement sur les risques associés à la sexualité: infections transmissibles sexuellement et par le sang, grossesse, usage du condom et prostitution (Roy et al., 2003; Haley et al., 2004; Agence de santé publique du Canada, 2006; Marshall et al., 2009). Or, en réduisant la complexité des expériences affectives et sexuelles à des comportements à risque pour la santé des jeunes, ces travaux tendent à présenter la rue comme une situation menaçante et dangereuse (Rayburn \& Corzine, 2010).

Dans une volonté de rompre avec cette vision de «dangerosité» de la situation de rue, certains travaux ont analysé le sens que ces jeunes donnent à leurs expériences affectives et sexuelles. Plusieurs de ces travaux montrent que la majorité des jeunes tissent peu de relations affectives et sexuelles en situation de rue et que $70 \%$ à $94 \%$ d'entre eux seraient célibataires (Fournier, 2001; Lussier et al., 2002; Loates $\&$ Walsh, 2010). Pour expliquer cette absence d'expériences affectives

3. Dans cet article, c'est le concept «d'expériences affectives et sexuelles» qui est utilisé pour rendre compte de la diversité des modèles d'intimité dans la société contemporaine et de la pluralité des liens entretenus avec les partenaires. En effet, certains auteurs proposent que les expériences affectives et sexuelles dans la société contemporaine se construisent dans un rapport égalitaire où les partenaires sont reconnus comme des personnes à part entière (Giddens, 1992; de Singly, 1996), tandis que pour d'autres, ces expériences s'établissent dans un rapport instrumental où les partenaires sont vus comme les objets d'une satisfaction personnelle (Sennett, 1979; Bauman, 2004).

4. Bien que le terme «jeunes de la rue» soit le plus souvent utilisé dans les écrits scientifiques, nous nous référerons dans cet article à la notion de «jeunes en situation de rue» afin de mettre en évidence le caractère dynamique et symbolique de cette réalité sociale. Plusieurs travaux montrent que les conditions de vie précaires et instables de la situation de rue ne sont pas vécues de la même façon par tous les jeunes (Parazelli, 2002; Bellot, 2005). Dans ce contexte, la rue n'est pas conçue comme un espace géographique ni un état statique, mais comme une situation de vie flexible et mouvante à laquelle les jeunes sont confrontés. 
et sexuelles, certains auteurs mettent de l'avant la précarité des conditions de vie en situation de rue (Côté, 1989; Lanzarini, 2000; Jamoulle, 2009). Par exemple, l'enquête de Lanzarini (2000), ancrée dans une approche néomarxiste, suggère que l'incertitude et l'hostilité provoquées par l'instabilité résidentielle et économique de la situation de rue conduisent les jeunes à se retirer de toute forme d'expériences affectives et sexuelles, retrait qualifié de «pauvreté sexuelle».

Bon nombre de ces études attribuent un caractère opportuniste et intéressé aux relations affectives et sexuelles des jeunes en situation de rue. Par exemple, Jamoulle (2009: 11) décrit les rencontres amoureuses des jeunes en situation de rue comme des «stratégies sociales et affectives». D'autres suggèrent que les expériences affectives et sexuelles de ces jeunes visent à combler un vide affectif (Levac \& Labelle, 2007), à briser l'isolement (Kidd \& Davidson, 2007), à fournir un soutien économique et psychologique (Kidd \& Davidson, 2007; Loates \& Walsh, 2010; Rayburn \& Corzine, 2010; Blais et al., 2012), ainsi qu'à les soutenir dans leur sortie du milieu de la drogue ou de la situation de rue (Kidd \& Davidson, 2007; Levac \& Labelle, 2007; Blais et al., 2012). Ces travaux mettent de l'avant une certaine instrumentalisation des liens affectifs et sexuels par les jeunes pour survivre en situation de rue.

Dans cette logique d'instrumentalisation, quelques études mettent en évidence que les jeunes en situation de rue, principalement les jeunes filles, ont recours aux transactions sexuelles ${ }^{5}$ afin de répondre soit à leurs besoins essentiels, comme se nourrir, se loger et se vêtir, soit à leur dépendance à la drogue (Lanzarini, 2000; Tyler \& Johnson, 2006; Kidd \& Davidson, 2007; Jamoulle, 2009; Watson, 2011). Certains de ces travaux montrent que les jeunes ont recours aux transactions sexuelles parce qu'ils ne voient pas d'autre alternative pour assurer leur survie (Tyler \& Johnson, 2006). Ces études tentent de se démarquer des travaux axés sur les risques, mais elles véhiculent une conception où la situation de rue est considérée comme une menace pour la santé ou la survie des jeunes. Cette lecture repose probablement sur le fait que l'on comprend mal comment les expériences affectives et sexuelles

5. Le concept de «transactions sexuelles» est utilisé dans cet article afin de désigner toutes les formes d'échange sexuel impliquant une compensation. Ce concept se veut plus neutre et plus englobant que les notions qui lui sont affiliées, comme celles de «prostitution», de «travail du sexe» ou de «commerce du sexe» qui ont tendance, selon LeclercMaadlada (2004: 3), à réduire l'échange sexuel à une compensation monétaire. 
des jeunes se construisent dans un rapport dynamique avec les conditions de vie de la situation de rue ${ }^{6}$.

Le cadre analytique de cette étude s'appuie sur la sociologie de l'expérience de Dubet (1994) et vise à articuler les interactions affectives et sexuelles des jeunes aux conditions de vie de la situation de rue. Dubet (1994) propose un champ théorique où la notion d'expérience est mise de l'avant pour rendre compte de l'articulation de l'univers subjectif des individus à leurs conditions de vie. Le concept d'expérience est défini comme les représentations, les émotions et les actions des acteurs au sein des «systèmes de relations et de représentations qui les fabriquent»(Dubet, 2007: 44). Selon cette perspective théorique, l'analyse des logiques d'action de l'expérience permet d'appréhender l'autonomie, la mouvance et la pluralité des réalités individuelles. La sociologie de l'expérience offre donc l'occasion d'analyser les relations affectives et sexuelles comme des expériences de vie qui témoignent de la résistance et de la volonté des jeunes à améliorer leurs conditions de vie en situation de rue.

Appliquée à l'objet d'étude des relations affectives et sexuelles en situation de rue chez les jeunes, la notion d'expérience permet de dégager trois concepts analytiques. Le premier, «l'expérience de rue», est défini comme les représentations, émotions et conduites que les jeunes entretiennent à l'égard de leur situation de rue. Le deuxième, «l'expérience affective et sexuelle», renvoie aux représentations, émotions et conduites que les jeunes entretiennent, ressentent et adoptent dans leurs relations affectives et sexuelles. Le troisième, «les expériences affectives et sexuelles en situation de rue», est défini comme les articulations entre les expériences de rue et les expériences affectives et sexuelles des jeunes. À la lumière de ce cadre analytique, cet article tente de comprendre comment les jeunes construisent leurs expériences affectives et sexuelles en situation de rue.

6. À notre connaissance, une seule équipe de recherche a étudié l'articulation entre le sens que les personnes donnent à leurs relations affectives et sexuelles et le sens qu'elles donnent à la situation de rue (Laporte et al., 2007; Pourette \& Oppenchaim, 2007; Oppenchaim et al., 2010). Si ces études mettent en relief différentes expériences affectives et sexuelles en situation de rue, elles présentent toutefois certaines caractéristiques méthodologiques (variabilité dans l'âge des participants, à savoir de 20 à 60 ans) et culturelles (étude réalisée en France) qui viennent limiter la transférabilité de ses constats à la réalité des jeunes en situation de rue à Montréal. 


\section{Une démarche méthodologique qualitative et typologique}

Le recrutement des participants a été réalisé d'octobre 2007 à avril 2010 à partir de deux stratégies. Premièrement, des encarts publicitaires ont été affichés au sein de différentes ressources pour jeunes en situation de rue à Montréal afin de publiciser le projet de recherche. Deuxièmement, inspiré de l'échantillonnage en boule de neige, les jeunes rencontrés ont été invités à transmettre la proposition de recrutement à leurs amis et connaissances en situation de rue. Les participants devaient répondre aux critères d'admissibilité suivants: 1) être âgé de 18 à 25 ans; 2) avoir été sans endroit où dormir au moins une fois durant la dernière année; et 3) avoir fréquenté des ressources pour jeunes en situation de rue au moins une fois durant la dernière année.

$\mathrm{Au}$ total, trente-deux jeunes en situation de rue $(18$ femmes, 14 hommes) âgés de 18 à 27 ans $^{7}$ (moyenne de 22 ans) ont été rencontrés. Une entrevue semi-dirigée d'environ une heure a été réalisée avec les participants à partir des dimensions suivantes: 1) les représentations de l'amour et de la sexualité; 2) les expériences amoureuses et sexuelles vécues; 3) les représentations de la situation de rue; 4) les expériences vécues en situation de rue; 5) les relations interpersonnelles en situation de rue; et 6) les représentations de l'avenir. Cette étude a reçu l'approbation éthique du Comité institutionnel d'éthique de la recherche de l'Université du Québec à Montréal. Le consentement libre et éclairé des jeunes a été assuré à l'aide d'un formulaire de consentement qui a été lu, discuté et signé par chacun des participants. Tous les noms ont été remplacés dans les retranscriptions par des prénoms fictifs. Un montant de $30 \$$ a été remis à chacun des participants à titre de dédommagement pour leur déplacement.

Le processus d'analyse des témoignages s'inspire de la démarche qualitative en deux étapes de Tesch (1990): la déconstruction et la reconstruction des données. Pour la déconstruction des données, une

7. Malgré le critère lié à l'âge, un jeune de plus de 25 ans (Benoit, âgé de 27 ans) a été inclus dans l'échantillon final. Comme le recrutement s'est élaboré selon l'échantillonnage théorique, c'est-à-dire qu'il s'est construit par étapes successives en réponse aux analyses des données et des lectures théoriques (Laperrière, 1997), le récit de Benoit s'est ajouté en raison de sa pertinence empirique quant à l'expérience de l'envahissement par la drogue. 
lecture verticale des entretiens a été réalisée afin de dégager le sens accordé par chacun des participants à leur expérience de rue et leur expérience affective et sexuelle. Ensuite, une fiche synthèse résumant l'articulation entre l'expérience de rue et l'expérience affective et sexuelle a été rédigée pour chacun des jeunes. Pour l'étape de la reconstruction des données, une analyse typologique (Schnapper, 2005 : 93) a été réalisée afin de dégager des types idéaux, c'est-à-dire de «grands ensembles conceptuels et schématiques qui permettent de rendre intelligibles les conduites et les discours observés». Il importe de préciser que des types idéaux ne constituent pas une représentation exacte des récits particuliers, mais une abstraction des expériences vécues. Selon Schnapper (2005), cette conception par types idéaux est pertinente pour rendre compte des expériences individuelles, car elle permet d'illustrer le rapport dynamique entre les conditions de vie et l'espace de liberté des acteurs. Pour la présente étude, l'analyse typologique n'a pas servi à dégager des trajectoires de vie typiques, mais elle a été utilisée pour identifier les articulations typiques entre les expériences de rue et les expériences affectives et sexuelles des jeunes. Concrètement, une classification des fiches synthèses a été effectuée à partir des ressemblances et des divergences quant à l'articulation entre les expériences de rue et les expériences affectives et sexuelles des jeunes. Finalement, cette classification a été suivie d'un travail de catégorisation conceptuelle qui a permis d'identifier les caractéristiques de ces différents types idéaux qui sont désignés ici comme les «figures des expériences affectives et sexuelles en situation de rue».

Il importe de tenir compte de certaines limites à cette étude, notamment celles associées au nombre réduit de l'échantillon. Étant donné que seulement trente-deux participants ont été recrutés pour cette recherche, les résultats dégagés ne peuvent être généralisés à l'ensemble de la population des jeunes en situation de rue. Également, devant le malaise de certains jeunes à discuter de leurs expériences affectives et sexuelles, les intervieweurs ont dû poser de nombreuses questions afin de relancer leur discours. Il est alors possible d'avancer que les jeunes rencontrés ont pu tenter de présenter un discours teinté par la désirabilité sociale en l'adaptant aux demandes implicites des chercheurs. 


\section{Les figures des expériences affectives et sexuelles en situation de rue}

L'analyse des témoignages a permis de dégager cinq figures des expériences affectives et sexuelles en situation de rue chez les jeunes: la valorisation de la réussite criminelle, le retrait, la survie, l'engagement et l'envahissement par la drogue. Pour illustrer chacune de ces figures, les témoignages de quelques jeunes ont été retenus à titre de récit typique.

\section{La figure de la valorisation de la réussite criminelle}

La figure de la valorisation de la réussite criminelle a été dégagée à partir du récit de quatre jeunes hommes âgés de 19 à 25 ans (moyenne de 22 ans). Si ces jeunes disent s'initier aux activités criminelles (principalement la vente de drogues) pour contrer les conditions de vie précaires de la situation de rue, ils y découvrent toutefois une expérience favorisant la construction d'une image sociale de réussite. L'intégration au milieu criminel leur procure non seulement une certaine autonomie économique, mais aussi une image sociale de réussite qui, selon ces jeunes, les place dans un rapport de domination à l'égard des autres jeunes en situation de rue.

J'étais «boss de rue», c'est moi qui fournissais les vendeurs de drogues. On pouvait dire que j'étais devenu quelqu'un! Quand je me promenais sur la rue, le monde me reconnaissait. Quand j'entrais dans le parc, le monde savait qui j'étais et que c'était mon parc. (Sébastien, 19 ans)

En réaction à leur intégration au milieu criminel, ces jeunes rapportent des expériences sexuelles sans engagement affectif pour à la fois tirer profit de leur image sociale de réussite et la consolider. Certains disent avoir rencontré de nombreuses partenaires sexuelles durant leur situation de rue en raison de leur intégration au milieu criminel, lequel, selon eux, leur a permis d'acquérir un certain pouvoir de séduction sur les jeunes femmes. D'après leurs propos, cette attirance s'explique par le fait que la vente de drogues leur procure une certaine stabilité financière et économique dont les jeunes femmes en situation de rue souhaitent bénéficier.

Je rencontrais des filles un peu partout, elles étaient contentes de mon service [de vente de drogues]... Je leur demandais si elles voulaient faire un petit tour chez mon ami avec moi (petit rire). Elles, ce qu'elles voulaient, 
s'étaient se coucher, alors je leur disais: «Ok, tu peux te coucher, mais après, essaye de prendre soin de moi.» Je ne voulais pas qu'elles s'en aillent sans que j'aie mon «nanane»! (François, 23 ans)

Ces participants disent fréquenter de belles et séduisantes jeunes femmes qui sont attirées par l'aura de la criminalité. Par ce discours sur l'importance de la beauté de leurs partenaires, les jeunes de cette figure semblent évoquer leur propre capacité à séduire les jeunes femmes les plus attirantes et convoitées. Par conséquent, il est possible de croire que la popularité auprès des jeunes femmes constitue un signe de réussite qui renforce leur image sociale.

Je n'amènerais plus une fille au centre-ville pour la présenter à mes chums (rire). Parce que quand tu arrives avec une belle femme, tu la présentes à tes chums, ils en veulent une pitoune eux autres aussi! [...] Quand tu sors avec LA pitoune, tous tes chums la veulent! (Sébastien, 19 ans)

\section{La figure du retrait}

La figure du retrait regroupe les témoignages de sept jeunes hommes âgés de 18 à 25 ans (moyenne de 21 ans). Si ces jeunes disent que la période de vie avant la situation de rue était empreinte de réussite sociale, ils mentionnent que la situation de rue est, à l'opposé, vécue comme une expérience disqualifiante et humiliante. Devant ce sentiment d'humiliation, ces jeunes se présentent comme des individus dévalorisés et amoindris.

Ça a été dégueulasse de se retrouver dans la rue, je n'avais plus d'argent... Il n'y avait plus rien autour de moi. Moi, [mon monde] c'est avec le gazon vert, les petites fleurs, les petits papillons, un bel après-midi, bien relax et rien de plus... Je n'étais pas à ma place. (Jack, 25 ans)

Face à ce sentiment d'humiliation provoqué par leur situation de rue, les jeunes de cette figure disent désinvestir les expériences affectives et sexuelles. Ils rapportent que l'humiliation associée à la situation de rue compromet leur pouvoir d'attractivité et de séduction. En réaction à l'image de l'homme pauvre et peu séduisant qu'ils disent projeter, ils préfèrent s'abstenir de tout contact avec de potentielles partenaires.

Ne pas avoir de logement et être dans la rue, comme je le suis en ce moment, j'aurais honte d'amener une copine ici. C'est une maison pour les jeunes dans la rue ici... J'aurais honte de l'amener, parce que j'ai honte moi-même d'être ici, de ne pas être dans un logement. (Alex, 22 ans) 
Plutôt que de s'investir dans des expériences affectives et sexuelles, ces jeunes racontent préférer concentrer leur attention sur la sortie de la situation de rue. Selon eux, le fait de développer et d'entretenir des expériences affectives et sexuelles les ancrerait davantage dans la situation de rue plutôt que de les aider à s'en sortir.

Je veux me sortir de là le plus tôt possible. C'est pour ça que ces temps-ci, les femmes, je ne fais que les regarder et rêver. [...] Il y en a plein des belles filles, sauf qu'elles ne sont pas pour moi... Elles sont dans les branches de la consommation et de la quête. Et moi, je veux décrocher du monde de la rue, je n'aime pas ça. (Christian, 24 ans)

\section{La figure de la survie}

La figure de la survie caractérise sept jeunes femmes âgées de 20 à 25 ans (moyenne de 22 ans). Ces jeunes rapportent une expérience de survie en raison de la précarité des conditions de vie en situation de rue. Elles disent mettre en place une diversité de stratégies pour répondre à leurs besoins essentiels, à savoir s'héberger, s'alimenter, se vêtir et se laver. Selon ces jeunes, cette précarité suscite beaucoup d'anxiété et d'inquiétude en raison de son caractère jugé dangereux et imprévu.

J'ai toujours eu peur de vivre dans la rue, car tu peux te faire tuer ou violer. C'est pour ça que je ne voulais pas coucher dehors. Quand tu es désespérée et que tu ne trouves pas d'autres solutions, alors tu vois l'hôpital le plus proche et tu te fais vomir [pour avoir une place à l'urgence]. (Jessica, 20 ans)

Ces jeunes rapportent établir des expériences affectives et sexuelles principalement sous la forme de transactions sexuelles pour répondre aux conditions de vie précaires de la situation de rue. En effet, les jeunes femmes dont les témoignages ont permis de dégager cette figure, à l'exception d'une seule ${ }^{8}$, ont indiqué avoir eu recours aux transactions sexuelles, à un moment ou un autre de leur situation de rue, en échange d'argent, d'hébergement, de nourriture et de vêtements.

Des fois, je ne savais pas où j'allais coucher et comme je ne voulais pas dormir dans la rue, alors je cherchais quelqu'un avec qui je pouvais m'en

8. Une jeune femme précise que, si elle n'a jamais eu recours aux transactions sexuelles, elle a déjà envisagé sérieusement cette pratique lorsqu'elle ne voyait pas d'autres solutions possibles pour obtenir de l'argent. 
aller. À la fin, je finissais par coucher avec cette personne-là. Mais je n'avais pas envie... (Allie, 21 ans)

Pour plusieurs de ces jeunes, c'est le poids des conditions de vie précaires en situation de rue qui fait obstacle au maintien d'expériences affectives. Si quelques-unes rapportent avoir vécu des relations amoureuses en situation de rue, la plupart d'entre elles témoignent du fait qu'elles n'ont ni le temps ni l'énergie pour s'investir dans une expérience affective. Selon elles, l'exigence de la survie fait en sorte qu'elles doivent répondre à leurs besoins personnels avant de répondre à ceux d'un partenaire. Dans ce contexte, l'amour est considéré comme un objectif à long terme associé à une stabilisation à l'extérieur de la situation de rue.

Je ne retomberais pas dans une relation, car je ne suis pas prête... Peut-être quand je serais bien installée, quand je vais être plus stable, moralement et physiquement. Quand je vais avoir accompli mes objectifs en appartement, à ce moment-là, ce sera possible de tomber en amour. (Jessica, 20 ans)

\section{La figure de l'engagement}

La figure de l'engagement a été dégagée à partir des témoignages de neuf jeunes femmes âgées de 18 à 25 ans (moyenne de 21 ans). Ces jeunes rapportent une expérience positive de la situation de rue, notamment en termes de liberté et de festivité, qui se traduit par un engagement contestataire envers le consumérisme. Pour ces jeunes, cet engagement anticonformiste prend la forme d'un véritable mode de vie au sens où le définit Laporte et al. (2007: 24), à savoir «des attitudes et des valeurs communes aux membres du groupe, ainsi définis et distingués d'autres collectifs».

Dans la rue, tu te construis une société, mais sans être une société, tu vois ce que je veux dire? On s'est construit une autre société qui veut évoluer et changer le monde. [...] Moi, je suis marginale, je ne suis pas conformiste. (Amélie, 20 ans)

Les jeunes de cette figure rapportent un investissement affectif et sexuel qui favorise leur intégration au mode de vie anticonformiste. Selon leurs propos, cet investissement prend la forme, entre autres, d'expériences affectives au sein desquelles leurs partenaires les aident à s'intégrer à la situation de rue. Plusieurs de ces jeunes mentionnent que leurs partenaires jouent le rôle de "partenaires de rue» avec lesquels elles apprennent les différents codes sociaux de la situation de rue. 
J'ai rencontré un gars quand j'avais 18 ans. J'ai été avec lui pendant deux ans et demi... Je commençais à être dans la rue, tandis que lui ça faisait des années qu'il était dans la rue. Moi je trouvais ça cool, je le trouvais hot. J'avais l'impression qu'il pouvait m'apprendre des affaires. [...] C'était mon meilleur ami, c'était mon partner de voyage, mon partenaire de vie! (Pauline, 22 ans)

Si ces jeunes rapportent de nombreuses activités sexuelles en situation de rue, toutes condamnent le recours aux transactions sexuelles. Selon elles, les transactions sexuelles sont considérées comme un acte irrespectueux de soi. Comme d'autres jeunes de cette figure, le témoignage d'Émilie fait voir que les transactions sexuelles vont à l'encontre du mode de vie anticonformiste des jeunes de cette figure, en plus de s'inscrire en faux contre leurs stratégies de débrouillardise au sein desquelles le réseau d'amis constitue une source d'entraide et de soutien.

Si je n'ai jamais fait de prostitution, c'est par respect pour moi-même... Mon cash, je peux le faire d'une autre manière que ça. Ça va prendre plus de temps, sauf que je ne vendrais pas mon corps pour de l'argent... Je n'envoie pas chier le monde qui le fait, parce que c'est leur décision, sauf que moi, je trouve ça dégueulasse. (Émilie, 18 ans)

\section{La figure de l'envahissement par la drogue}

Cette figure a été identifiée sur la base de quatorze témoignages: 8 femmes et 6 hommes, âgés de 19 à 27 ans (moyenne de 23 ans). Si les jeunes des autres figures disent concevoir la drogue comme une expérience récréative, pour les jeunes de cette figure, la consommation de drogue représente une expérience envahissante qui prend le dessus sur l'ensemble de leurs activités quotidiennes. Ils disent négliger la gestion de leurs besoins essentiels au profit d'une recherche quotidienne d'argent nécessaire à payer leur consommation de drogues.

J'ai déjà volé mes parents pour de la drogue. Je les aime mes parents, mais je les ai volés et je les ai crossés. Parce que dans la rue, la drogue prend le dessus sur tout! Je n'avais plus le contrôle, je n'étais pas assez fort psychologiquement, pas assez fort pour dire que la drogue ne prendra pas le contrôle sur moi. (Benoît, 27 ans)

En réaction à l'envahissement par la consommation de drogues, les jeunes de cette figure rapportent un investissement de l'expérience sexuelle pour répondre à leur dépendance aux substances. Dans ce 
contexte, ces jeunes mentionnent faire usage de transactions sexuelles pour obtenir soit de la drogue, soit l'argent nécessaire à se la procurer.

Moi, c'est mon problème de toxicomanie qui m'a apporté à la prostitution. Je n'irais pas faire ça pour me payer un bateau, des études ou n'importe quoi. Dans mon cas, la prostitution, c'était vraiment pour la consommation, la déchéance, l'autodestruction. (Lili, 24 ans)

Pour ces jeunes, la consommation de drogues en situation de rue et son rapport aux transactions sexuelles rendent peu propice le maintien d'expériences affectives. Leur discours met en évidence que l'envahissement par la drogue vient monopoliser tout le temps et l'énergie des jeunes, ce qui fait obstacle à un investissement affectif auprès d'une autre personne.

Je sortais avec une fille pendant que j'étais dans la rue et je sentais une certaine émotion pour elle, mais, dans ce temps-là, je m'injectais de la drogue. Comme je consommais beaucoup, même si j'éprouvais quelque chose pour elle, j'étais plus porté à aller vers ma drogue que de rester avec ma blonde... C'est la consommation de drogues qui me «drivait» [dirigeait]! (Benoît, 27 ans)

\section{Discussion}

L'objectif de cette étude était de comprendre, à partir du point de vue des jeunes, comment se construisent leurs expériences affectives et sexuelles en situation de rue. Cinq figures ont été dégagées: la valorisation de la réussite criminelle, le retrait, la survie, l'engagement et l'envahissement par la drogue. Une réflexion sur le croisement entre ces différentes figures permet de dégager deux rapports distincts quant à la construction des expériences affectives et sexuelles en situation de rue.

Premièrement, les figures de l'engagement et du retrait évoquent une "expérience subjectivée», c'est-à-dire un engagement affectif et émotionnel avec un partenaire conçu comme un sujet à part entière. À l'instar des travaux de Giddens (1992) et de Singly (1996), ces jeunes évoquent l'importance des rapports égalitaires et réciproques avec leurs partenaires pour la création d'un espace commun de partage affectif et sexuel. Par exemple, pour les jeunes de la figure de l'engagement, les partenaires leur permettent de développer un sentiment d'appartenance avec d'autres jeunes en situation de rue et, ainsi, de se sentir reconnus 
et appréciés par des pairs qui partagent une réalité similaire à la leur. Pour les jeunes de la figure du retrait, ils ne veulent pas s'engager dans des expériences affectives et sexuelles parce qu'ils ne se sentent pas à la hauteur d'une partenaire potentielle. Contrairement à certains travaux qui voient les expériences affectives et sexuelles comme une passerelle vers la sortie de rue (Kidd \& Davidson, 2007; Levac \& Labelle, 2007), ces jeunes rapportent que l'engagement avec une partenaire potentielle constitue plutôt une menace d'enfermement en situation de rue. Ces deux figures font la démonstration que les partenaires en situation de rue ne se réduisent pas à des instruments de survie, mais peuvent aussi être conçus comme des sujets participant à la construction d'une relation égalitaire, réciproque et potentiellement émancipatoire.

Deuxièmement, les témoignages des jeunes des figures de la valorisation de la réussite criminelle et de la survie traduisent une «expérience objectivée» caractérisée par un désengagement affectif et émotionnel avec un partenaire conçu comme un instrument de sa satisfaction personnelle. À l'instar de Sennett (1979) et de Bauman (2004), les partenaires de ces jeunes ne sont pas considérés comme des individus à part entière, mais plutôt comme des sources de satisfaction matérielle (hébergement, nourriture et vêtements) et symbolique (reconnaissance et position sociale). Ce constat rejoint d'autres travaux qui mettent en évidence que les jeunes se voient contraints d'instrumentaliser leurs partenaires pour leurs propres besoins (Kidd \& Davidson, 2007; Levac \& Labelle, 2007; Jamoulle, 2009; Loates \& Walsh, 2010; Rayburn \& Corzine, 2010). À l'opposé de l'expérience subjectivée, il ne s'agit pas ici d'expériences affectives et sexuelles basées sur des principes d'égalité et de réciprocité, mais sur une marchandisation de la sexualité à des fins personnelles. En reprenant l'analyse sociologique de Bauman (2004: 27), il est possible de croire que ces figures traduisent des expériences affectives et sexuelles «liquides», c'est-à-dire qu'elles s'inscrivent dans une logique de consommation caractérisée par un «investissement rentable» et une «instantanéité et une jetabilité incarnées». Les partenaires de ces jeunes semblent utiles aussi longtemps qu'ils répondent à leurs besoins personnels, mais dès qu'ils ne remplissent plus leur fonction, ils peuvent se voir délaissés pour d'autres partenaires jugés plus satisfaisants. Les témoignages de ces jeunes font donc voir que les partenaires sont vus prioritairement comme des objets, notamment en raison du poids des conditions de vie en situation de rue, qui les font espérer pouvoir en tirer un certain profit. 
Quant aux jeunes qui se retrouvent dans la figure de l'envahissement par la drogue, l'analyse montre que la majorité d'entre eux (9/14) se sont retrouvés, à un moment où un autre de leur situation de rue, dans une autre figure. En dépit de l'absence de mouvance entre les quatre autres figures, celle de l'envahissement par la drogue permet, quant à elle, de constater un déplacement important au sein des expériences de vie des participants. Comme l'illustre la figure 1, cette mouvance s'explique par un changement dans l'importance que prend la consommation de drogues chez ces jeunes. Cet envahissement n'est pas sans rappeler ce que Castel (1998: 25) décrit comme l'«expérience totale» de consommation de drogues, c'est-à-dire «la recherche et la consommation du produit qui parasitent l'ensemble des relations au monde d'un individu et mettent toute sa vie au service de cette passion». D'une expérience récréative au sein des autres figures, la consommation de drogues devient une expérience totale qui fait en sorte de reléguer au second plan les expériences de survie, de valorisation de la réussite criminelle, d'engagement et de retrait de ces jeunes. Comme d'autres travaux l'ont déjà montré (Lanzarini, 2000; Tyler \& Johnson, 2006; Kidd \& Davidson, 2007 ; Jamoulle, 2009; Watson, 2011), ces jeunes se voient pousser vers une marchandisation de la sexualité afin de répondre à leur dépendance aux substances. Si les jeunes de la figure de l'envahissement par la drogue rapportent établir des expériences affectives et sexuelles centrées sur l'objectivation de leurs partenaires, ils ne le font pas pour répondre aux conditions de vie précaires de la situation de rue, comme c'est le cas pour les jeunes de la figure de la survie, mais pour répondre prioritairement à leurs besoins de consommation de drogues. En d'autres mots, les expériences affectives et sexuelles ne sont pas considérées par ces jeunes comme une expérience de plaisir, d'engagement affectif ou de subsistance, mais comme un instrument au service de leur consommation envahissante de drogues.

À l'instar de l'analyse de Lanzarini (2000), les figures identifiées semblent témoigner d'expériences affectives et sexuelles différenciées selon le genre. Il est possible de croire que la situation de rue offre une opportunité pour certains jeunes d'adhérer aux rôles de genre, à savoir les caractéristiques socialement assignées aux hommes et aux femmes. Par exemple, pour certains jeunes hommes, l'intégration au milieu criminel semble les amener à valoriser des expériences empreintes de performance sexuelle et de domination à l'égard des femmes, tandis que pour certaines jeunes femmes, l'appartenance au mode de vie 
F I G URE 1

Mouvance entre les figures des expériences affectives et sexuelles en situation de rue

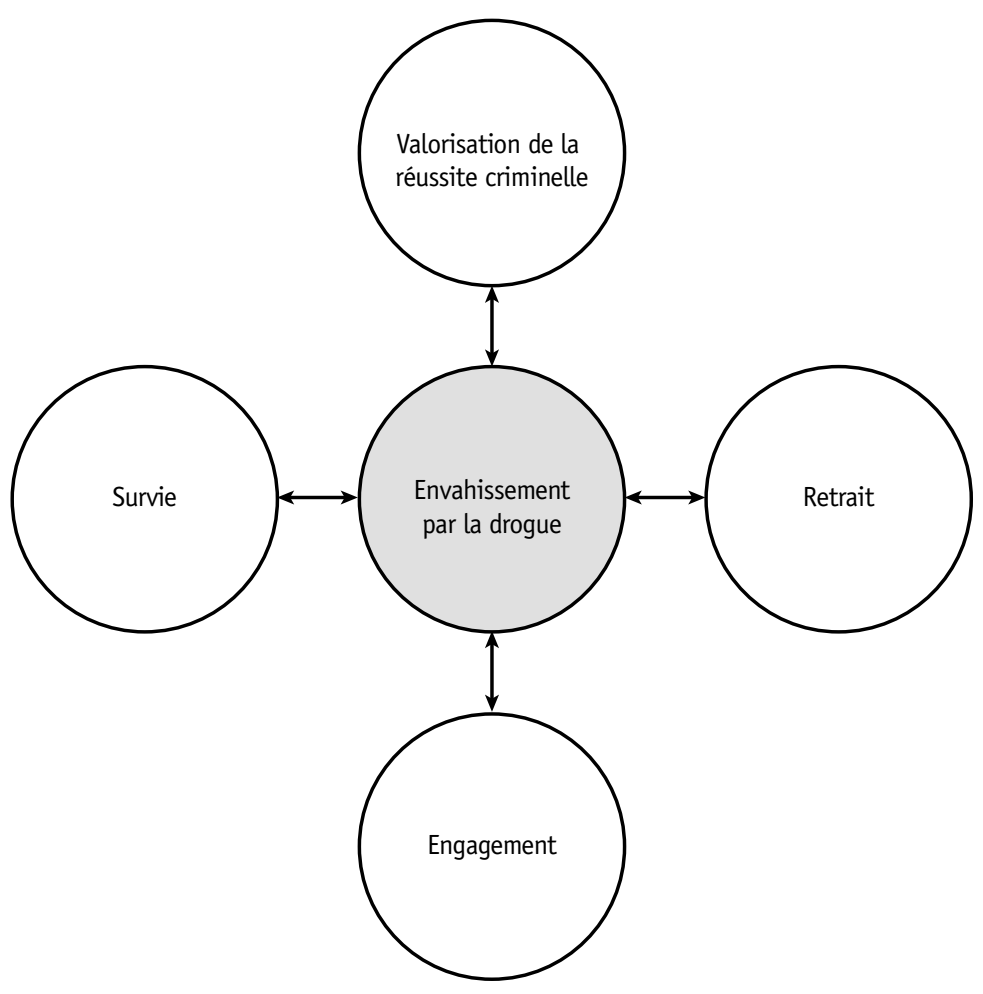

anticonformiste semble les conduire à développer des relations basées sur l'affection et la tendresse. Par contre, comme le suggèrent Jamoulle (2009) et Oppenchaim et al. (2010), il est possible de croire que l'expérience totale de la consommation de drogues en situation de rue vient parasiter l'adhésion aux rôles de genre. L'important pour les jeunes inscrits dans la figure de l'envahissement par la drogue ne semble pas de répondre aux caractéristiques masculines ou féminines prescrites par la société, mais de répondre par tous les moyens possibles, et indépendamment du genre, à leur dépendance aux substances. 


\section{Conclusion}

Cette étude a permis d'identifier une pluralité d'expériences affectives et sexuelles en situation de rue chez les jeunes à Montréal. À l'instar de Rayburn et Corzine (2010), cette analyse permet de déconstruire une lecture homogène de la situation de rue chez ces jeunes. Par le recours à la sociologie de l'expérience (Dubet, 1994), il a été possible de mettre en évidence la façon dont les jeunes construisent eux-mêmes leurs expériences affectives et sexuelles, et ce, à l'intérieur des conditions de vie de la situation de rue. En effet, par l'analyse de leurs témoignages, cette recherche a permis de dresser un portrait des différentes stratégies que ces jeunes mettent en place pour se débrouiller et se construire en situation de rue. Cette étude permet de voir que les conditions de vie de la situation de rue influencent les actions et les significations des jeunes, mais ne constituent pas un poids inaltérable, car elles témoignent également de stratégies pour améliorer leur situation de vie. Par conséquent, il est possible de conclure que ces jeunes, en dépit de leurs conditions de vie précaires, ne sont pas différents des autres individus de la société. Comme tout un chacun, ces jeunes mobilisent ou rejettent les expériences affectives et sexuelles afin de se construire comme des individus à part entière de la société. Il serait intéressant, pour les futurs travaux de recherche, de comprendre davantage comment le temps passé en situation de rue et les cadres socionormatifs de genre peuvent influencer les expériences affectives et sexuelles de ces jeunes.

\section{Références}

Agence de santé publique du Canada. (2006). Les jeunes de la rue au Canada. Constatations découlant d'une surveillance accrue des jeunes de la rue au Canada 1999-2003. Ottawa: Agence de santé publique du Canada.

Bauman, Z. (2004). L'amour liquide. De la fragilité des liens entre les hommes. Rodez: Le Rouergue Chambon.

Bellot, C. (2005). La diversité des trajectoires de rue des jeunes à Montréal. In N. Brunelle, \& M.-M. Cousineau (eds), Trajectoires de déviance juvénile: les éclairages de la recherche qualitative (71-95). Sainte-Foy: Presses de l'Université du Québec.

Blais, M., Côté, P.-B., Manseau, H., Martel, M., \& Provencher, M.-A. (2012). Love among the homeless: A portrait of romantic and couple relationships among street-involved young adults in Montreal. Journal of Youth Studies, 15 (4), 403-420. 
Castel, R. (1998). Les sorties de la toxicomanie. Suisse: Éditions Universitaires Fribourg.

Côté, M.-M. (1989). Fuite et stratégie de survie des jeunes de la rue à Montréal. Santé mentale au Québec, 14 (2), 150-157.

Dubet, F. (1994). La sociologie de l'expérience. France: Éditions du Seuil.

Dubet, F. (2007). L'expérience sociologique. Paris: La Découverte.

Fournier, L. (2001). Enquête auprès de la clientèle des ressources pour personnes itinérantes des régions de Montréal-Centre et de Québec, 1998-1999. Québec: Institut de la statistique du Québec.

Giddens, A. (1992). La transformation de l'intimité. Sexualité, amour et érotisme dans les sociétés modernes. Rodez: Le Rouergue/Chambon.

Haley, N., Roy, E., Leclerc, P., Boudreau, J.-F., \& Boivin, J.-F. (2004). HIV risk profile of male street youth involved in survival sex. Sexually Transmitted Infections, 80, 526-530.

Jamoulle, P. (2009). Fragments d'intime. Amours, corps et solitudes aux marges urbaines. Paris: La Découverte.

Kidd, S. A., \& Davidson, L. (2007). "You have to adapt because you have no other choice": The stories of strength and resilience of 208 homeless youth in New York and Toronto. Journal of Community Psychology, 35 (2), 219-238.

Lanzarini, C. (2000). Survivre dans le monde sous-prolétaire. Paris: Presses universitaires de France.

Laperrière, A. (1997). La théorisation ancrée (grounded theory): démarche analytique et comparaison avec d'autres approches apparentées. In J. Poupart, J.-P. Deslauriers, L. Groulx, A. Laperrière, R. Mayer, \& A. Pires (eds.), La recherche qualitative: enjeux épistémologiques et méthodologiques (309-340). Boucherville: Gaëtan Morin.

Laporte, A., Le Méner, E., Oppencheim, N., Pourette, D., \& Carpentier, S. (2007). Survivre ou faire l'amour? La pluralité des expériences affectives et sexuelles des personnes sans-domicile-fixe. France: Rapport à l'Institut national de prévention et d'éducation pour la santé (INPES).

Leclerc-Madlada, S. (2004). Transactional sex and the pursuit of modernity. University of Cape Town: Centre for Social Science Research, Social Surveys Unit. Consulté le 10 octobre 2012, www.cssr.uct.ac.za/sites/cssr.uct.ac.za/ files/pubs/wp68.pdf

Levac, C., \& Labelle, F. (2007). La rue, un chemin tracé d'avance? Montréal: Le Refuge des jeunes de Montréal.

Loates, M., \& Walsh, C. A. (2010). Women negotiating sexual identity in the face of homelessness: From silence to satisfaction. Culture, Health $\mathcal{G}$ Sexuality, 12 (1), 87-101.

Lussier, V., Letendre, R., Morval, M., Pelletier, A., Poirier, M., Michaud, P., \& Gilbert, S. (2002). La quête au cœur de l'absence: les réseaux relationnels de jeunes adultes itinérants. Revue québécoise de psychologie, 23 (3), 79-103.

Marshall, B. D. L., Kerr, T., Shoveller, J. A., Qi, J., Montaner, J. S. G., \& Wood, E. (2009). Structural factors associated with an increased risk of HIV and sexually transmitted infection transmission among street-involved youth. BMC Public Health, 9 (7), 1-9. 
Oppenchaim, N., Pourette, D., Le Méner, E., \& Laporte, A. (2010). Sexualité et relations affectives des personnes sans domicile fixe. Entre contraintes sociales et parcours biographiques. Sociologie, 3 (1), 375-391.

Parazelli, M. (2002). La rue attractive: parcours et pratiques identitaires des jeunes de la rue. Sainte-Foy: Presses de l'Université du Québec.

Pourette, D., \& Oppenchaim, N. (2007). Améliorer les conditions de vie affective et sexuelle des SDF. La Santé de l'homme, 392, 42-43.

Rayburn, R. L., \& Corzine, J. (2010). Your shelter or mine? Romantic relationships among the homeless. Deviant Behavior, 31, 756-774,

Roy, É., Haley, N., Leclerc, P., Cédras, L., Weber, A., Claessens, C., \& Boivin, J.-F. (2003). HIV incidence among street youth in Montreal, Canada. AIDS, 17, 1071-1075.

Schnapper, D. (2005). La comprébension sociologique. Démarche de l'analyse typologique. Paris: Presses universitaires de France.

Sennett, R. (1979). Les tyrannies de l'intimité. Paris: Éditions du Seuil.

Singly, F. de (1996). Le soi, le couple et la famille. Paris: Nathan.

Tesch, R. (1990). Qualitative research: Analysis types and software tools. New York: The Falmer Press.

Tyler, K. A., \& Johnson, K. A. (2006). Trading sex: Voluntary or coerced? The experiences of homeless youth. Journal of Sex Research. 43 (3), 208-216.

Watson, J. (2011). Understanding survival sex: Young women, homelessness and intimate relationships. Journal of Youth Studies, 14 (6), 639-655.

ABTRACT - This study aims to understand how street young construct their affective and sexual experiences. Individual interviews were conducted with thirty-two street youths in Montreal (18 women, 14 men) aged from 18 to 27 years old. The testimonies were analyzed from a typological method (Schnapper, 2005) that identified five groups of affective and sexual experiences in the street context: criminal success, withdrawal, survival, commitment and avoidance through drugs. The analysis shows that youth from the withdrawal and the commitment groups reported an affective engagement with their intimate partners, while the youth from the criminal success and the survival groups described an instrumentalization of their intimate partners. The 'avoidance through drugs' group shows the influence of drugs, which push young people to instrumentalize sexuality in order to satisfy their addiction. This study helps to nuance the homogeneous and threatening conception of the street context by showing the different ways in which the youths mobilized their affective and sexual experiences in order to improve their situation.

KEYWORDS - Street youth, love, sexuality, typology, qualitative research.

RESUMEN - El presente estudio tiene como objetivo comprender como los jóvenes construyen sus experiencias afectivas y sexuales en situación de calle en Montreal. Se han realizado treinta y dos entrevistas individuales a jóvenes en situación de calle (18 mujeres y 14 varones) de entre 18 y 27 años. Los testimonios han sido analizados a partir de un método tipológico (Schnapper, 2005) que ha permitido identificar cinco figuras de experiencias afectivas y sexuales en situación de calle: la valorización del 
éxito criminal, la retirada, la supervivencia, el compromiso y la invasión de la droga. El análisis de los testimonios demuestra que los jóvenes de las figuras de la retirada y del compromiso se involucran afectivamente con sus parejas, mientras que las figuras de la valorización del éxito criminal y de la supervivencia, mas bien, describen una instrumentalización de sus parejas. La figura de la invasión de la droga, ilustra la influencia que tiene el consumo de drogas, empujando a los jóvenes hacia una mercantilización de la sexualidad para subvenir a su dependencia a las sustancias. Por lo tanto, este artículo permite de matizar una concepción homogénea y amenazante de la situación de calle, mostrando como los jóvenes movilizan de diversas maneras sus experiencias sexo-afectivas para mejorar su condición de vida.

PALABRAS CLAVE - Jóvenes de la calle, amor, sexualidad, tipología, investigación cualitativa. 